\title{
Fermi pseudo-potential and energy-dependent point interactions in one dimension
}

\author{
L. Tomio*, F. A. B. Coutinho ${ }^{\dagger}$, Y. Nogami** and F. M. Toyama ${ }^{*}$ \\ *Instituto de Física Teórica, UNESP, 01405-900, São Paulo, SP, Brazil \\ $\dagger$ Faculdade de Medicina, Universidade de São Paulo, 01246-903, São Paulo, SP, Brazil \\ ** Department of Physics and Astronomy, McMaster University, Hamilton, Canada L8S 4M1 \\ ${ }^{*}$ Department of Information and Communication Sciences, Kyoto Sangyo University, \\ Kyoto 603-8555, Japan
}

\begin{abstract}
There are point interactions in one dimension that can be interpreted as self-adjoint extensions (SAEs) of the kinetic energy [KE] operator. Here, we report the results obtained in two recent papers cited in [1]. In the first, we consider point interactions in one dimension in the form of the Fermi pseudo-potential, in one and two-channel cases. In the second, we consider a new type of point interactions that are self-adjoint and effectively energy-dependent.
\end{abstract}

There are point interactions in one dimension that can be interpreted as self-adjoint extensions (SAEs) of the kinetic energy [KE] operator [1]. Following [2] we examined point interactions in one dimension in the form of the Fermi pseudo-potential, considering one and two-channel cases. In the one-channel case, the Fermi pseudo-potential contains three real parameters. The SAEs also contain, apart from a trivial parameter $\theta$, three real parameters. We have found explicit relations between the three parameters of the pseudo-potential and those of the SAEs [3]. In the two-channel case the pseudopotential provides us with more general SAEs than obtained earlier [4]. The two-channel pseudo-potential can have ten parameters, which means that the pseudo-potential can represent a ten-parameter family of SAEs. If the pseudo-potential is the only interaction that is contained in the Hamiltonian of the system, time-reversal invariance holds. If we impose space symmetry the number of the parameters is reduced from ten to seven. The corresponding number of the parameters that was obtained in [4] is five. When timereversal invariance holds, the $S$-matrix of a two-channel system in one dimension can be expressed in terms of a $4 \times 4 K$-matrix that is real and symmetric. The $S$-matrix has ten real parameters. This is so irrespective of the form of the interaction as long as it conforms to time-reversal invariance. It is interesting that the number of the parameters of the $S$-matrix and that of the SAEs coincide.

As advocated in [2] the two-channel pseudo-potential can be a very powerful tool with which one can construct models of quantum memory, an essential component of quantum computing.

\footnotetext{
${ }^{1}$ Work supported by the Brazilian agencies FAPESP and CNPq, the NSERC of Canada and by the Ministry of Education, Culture, Sports, Science and Technology of Japan
} 
We are also considering a new type of point interactions in one dimension, characterized by boundary conditions at the origin that involve the second and/or higher order derivatives of the wave function. The interactions are self-adjoint and effectively energydependent [5]. A point interaction with constant boundary conditions can suport one or two bound states $[3,6]$. However, if we allow the interactions to be energy dependent, we can have infinite varieties of point interactions in one dimension. In this way, we can construct interactions that can support any number of bound states.

\section{REFERENCES}

1. S. Albeverio, F. Gesztesy, R. Høegh-Krohn H. and H. Holden, Solvable Models in Quantum Mechanics, Berlin: Springer, 1987.

2. T. T. Wu and M. L.Yu, J. Math. Phys. 43, 5949 (2002).

3. F. A. B. Coutinho, Y. Nogami, L. Tomio and F. M. Toyama, to appear in J. Phys. A: Math. Gen. (2004).

4. F. A. B. Coutinho, Y. Nogami and F. M. Toyama, J. Phys. A: Math. Gen. 37, 2989 (2004).

5. F. A. B. Coutinho, Y. Nogami, L. Tomio and F. M. Toyama, in preparation.

6. F. A. B. Coutinho, Y. Nogami and L. Tomio, J. Phys. A: Math. Gen. 32, 4931 (1999). 
Copyright of AIP Conference Proceedings is the property of American Institute of Physics and its content may not be copied or emailed to multiple sites or posted to a listserv without the copyright holder's express written permission. However, users may print, download, or email articles for individual use. 